\title{
Design of Custom Made Prosthesis of the Hip
}

\author{
Slobodan TABAKOVIĆ, Milan ZELJKOVIĆ, Zoran MILOJEVIĆ, Aleksandar ŽIVKOVIĆ
}

\begin{abstract}
Replacement of a natural hip joint with an artificial is one of the most commonly used surgical procedures in orthopaedic surgery. This paper describes the methodology of the hip joint prosthesis design, which presents the foundation for the automation of all stages of the development of custom-made prostheses. A general mathematical model based mostly on B-spline surfaces is used in order to define the prosthesis geometry. In order to verify the method, a software solution was developed and used for designing prostheses based on ten diagnostic images obtained by various methods. The results of the analysis of the ten designed prostheses, out of which five manufactured, show that deviations from the desired values are less than the errors caused by diagnostic imaging.
\end{abstract}

Keywords: Computer Aided Design; custom-made endoprosthesis; hip joint

\section{INTRODUCTION}

The surgical procedure of replacement of the natural joint of the hip with artificial one and establishment of the patient's lower limb function is one of the most commonly used procedures in orthopedic surgery. Research shows that over 500,000 procedures of this type are carried out every year in the US and UK alone [1]. As a consequence of the modern way of life, a significant increase in the number of diseases whose treatment requires replacement of the hip joint with an artificial one is expected in the near future [2].

A large number of studies in the field of hip orthopaedics aim at increasing the endoprosthesis' lifetime and the comfort of its use by studying the mechanisms of postoperative complications and using these findings in the design process. Thus far, loosening studies indicate the importance of the dimensions of hip endoprosthesis [3], applied materials [4] and body characteristics of patients [5]. Another significant cause of failures, fatigue fractures, may happen due to inadequate endoprosthesis [6] and tribological processes [7]. In addition, inadequate dimensions and materials choice may make unpleasant changes for patients in terms of the hip joint squeaking [8]. This points to the fact that the causes of failures can be related to the shape and dimensions of the artificial hip, choice of the type [9], and material structure [10] as well as tribological processes $[11,12]$.

Based on the results of these studies, it can be concluded that improvements in the hip endoprostheses' characteristics can be achieved by using a personalised approach to design, computer supported analyses in order to optimise the characteristics [13] and production. Recent studies indicate that the custom made endoprostheses allow considerably improved fittings of implants to the bone [14] and biomechanical characteristics [15] as well as significantly lower failure occurrences [16]. However, their application in clinical conditions was only possible in the last few years after the perfecting of the tomographic image segmentation process allowed the reconstruction of the pelvis region [17] and the definition of parametric CAD models of the bone [18], followed by the application of technologies that enable the production of implants in an acceptable time period using subtractive [9], or additive, [19] technologies.
This paper presents the original methodology of designing the prosthesis of the hip joint according to the patient's measurements. It is based on the following assumptions: for the automation of the design process of the body of endoprosthesis of the hip joint, it is appropriate to apply a general geometric model based on the mathematical description of the outer surface of the endoprosthesis using NURBS surfaces, and that arranging the sequence of defining the parameters describing the model allows flexible defining of correlations primarily according to the geometry of the femur, as well as characteristics of diseases, materials and tribological processes in the body.

For the purpose of verification of the procedure, a specialised software solution was developed based on which a number of hip endoprostheses were designed and developed.

\section{MATERIALS AND METHODS \\ 2.1 Basic Geometric Elements of Morphological Endoprosthesis According to Patient's Characteristics}

The shape and dimensions of hip joint endoprostheses depend on a number of parameters, among which the most important are: type and severity of the disease, age of the patient, morphological characteristics of the patient's femur and pelvis, to name but a few. By improving the implants' structure as well as the surgery itself, a large number of different types of endoprostheses were developed, which are categorised by the international standard ISO 7206-1.

Structurally, hip joint endoprostheses contain two components (Fig. 1): acetabular and femoral.

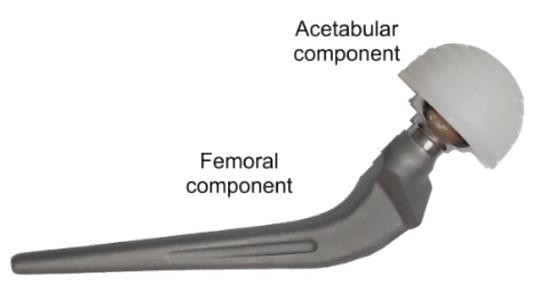

Figure 1 Hip joint endoprosthesis

The first makes an artificial hip joint that forms the connection between the femoral bone and the pelvis. The second, femoral part (the "body" of endoprosthesis) is 
making a connection between the artificial joint of the hip and the femoral bone.

The acetabular component (Fig. 2) consists of artificial femoral head, acetabular liner and shell.

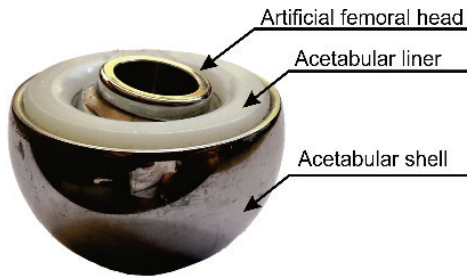

Figure 2 The acetabular component of the hip endoprosthesis

The choice of the acetabular component is restricted by the morphology of the pelvic region as well as the characteristics of the disease.

The body of the hip joint endoprosthesis is, from a geometric and functional point of view, a complex unity consisting of three segments of different shape and role in the artificial hip joint. According to the position in the femur, the segments are divided into distal, medial and proximal [20] (Fig. 3).

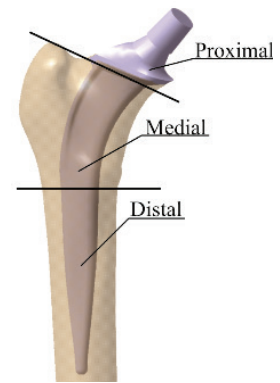

Figure 3 Segments of the body of the endoprosthesis

The distal segment plays a role in positioning the body of endoprosthesis into the medullary canal and forming the final bond strength between the endoprosthesis and femur.

The medial segment of the body of endoprosthesis is the part that follows the shape of the medullary canal and the line of the femur neck. The geometric shape that describes this segment consists of a complex surface, which by satisfying certain criteria depends on the geometry of the femur.

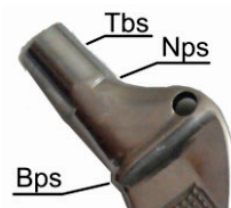

Figure 4 Proximal segment of the endoprosthesis

The proximal segment of the body of endoprosthesis is structurally the most complex part of the artificial hip. It contains a geometric form that replaces the removed neck of the femur and provides a connection to the artificial hip joint. The proximal segment consists of (Fig. 4):

- $\quad$ Supporting part (Bps). The basic role of this part is to provide an appropriate support for endoprosthesis.

- Proximal segment neck (Nps) replaces the natural neck of the femur and ensures the proper distance between the femur and the centre of the hip joint (offset).
- Connection Element (Tps). It provides a link between the artificial femoral head and the body of endoprosthesis, which is most often made in the form of a cone.

\subsection{FTN-Custom Hip Design Method}

As a result of the analysis of the morphological, kinematic and dynamic characteristics of the femoral bone and hip joint, research carried out in the previous period resulted in a large number of geometric parameters relevant to the definition of the endoprosthesis body [21, 14]. Depending on the type of endoprosthesis, ISO 7206-1 standard defines a different number of geometric parameters that can be used to characterise certain type. The development of custom made endoprosthesis involves a significantly greater number of parameters because, in addition to the basic objectives of the surgical procedure (restoring the function of the diseased hip), further objectives are included, such as reduced invasiveness of the procedure and patient recovery time.

The methodology of hip joint endoprostheses development using computer support includes a systematic approach to defining geometric parameters which are grouped according to their importance regarding the shape and function of the prosthesis. The custom hip design methodology is based on the application of three sets of parameters for defining the geometry of the endoprosthesis body. According to the purpose and stage in which they are used, the parameters can be categorised by their effect on: location and overall dimensions, orientation, and the shape of endoprosthesis (Fig. 5).

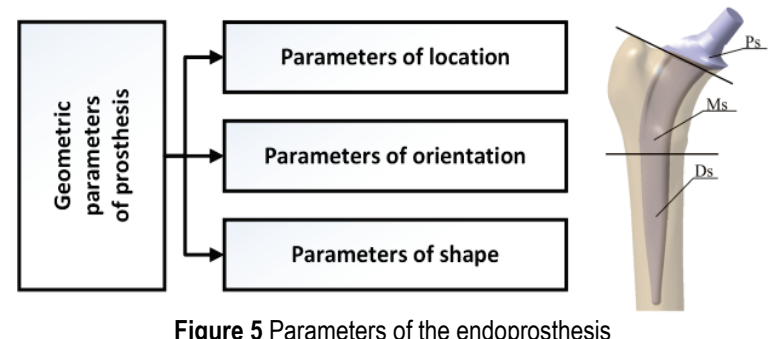

Methods for defining endoprosthesis parameters may vary depending on the type of diagnostic imaging. Parameters that describe endoprosthesis geometry are the consequence of the femur's morphology, nature and stage of the disease, implementation procedure and endoprosthesis' materials, to name but a few. The abbreviation FTN in the name of the method represents a local acronym for the Faculty of Technical Sciences, where the idea of modifying the geometry of endoprostheses using the categorised parameters was created.

\subsubsection{Parameters of Location and Overall Dimensions of Endoprosthesis}

The first phase of endoprosthesis design covers the definition of basic geometric parameters that determine the overall dimensions and the optimal position of endoprosthesis after implementation in the skeletal system. In this way, by having the goal in mind (restoring the geometry of the hip joint), the foundation for defining the elements of endoprosthesis is created. 
Location parameters are determined by the morphology of the femur and the criteria governed by the disease (Fig. 6).

The centre of the femoral head represents the point around which the femoral bone rotates in the hip joint. The position and dimensions of the femoral head are utilised in the dimensioning of the artificial hip joint, which is typically manufactured in different sizes. These dimensions are obtained by determining the centre of the sphere which describes the cloud of points representing the femoral head on the processed diagnostic image. This can be done by applying the least squares method to the sphere equation, for a set of u points which describe the entire femoral head or one of its parts.

$$
\sum_{i=1}^{u} e^{2}=\sum_{i=1}^{u}\left\{\sqrt{\left(x_{i}-x_{c}\right)^{2}+\left(y_{i}-y_{c}\right)^{2}+\left(z_{i}-z_{c}\right)^{2}}-r\right\}^{2}
$$

The unknown values of $r, x_{c}, y_{c}$ and $z_{c}$ are calculated from the system of equations obtained by equalling the partial first derivatives of Eq. (1) for all variables to zero.

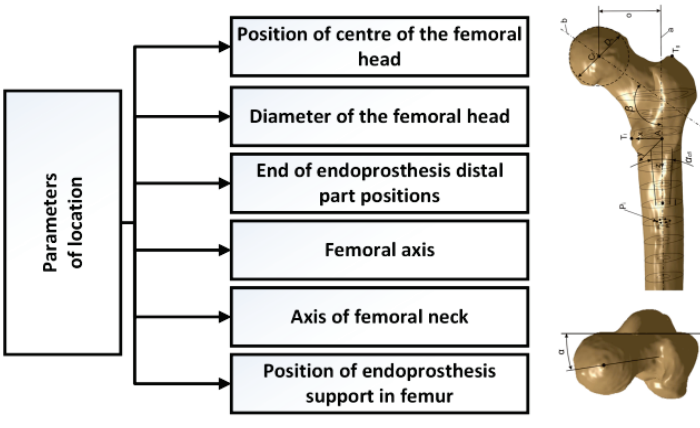

Figure 6 Endoprosthesis parameters of location

If the input data are in the form of a planar diagnostic image, femoral head information is obtained by approximating the points with a circle and subsequently determining its centre.

Anatomic axis of the femur is a linear function that describes the line of the femoral canal. This rule is obtained by using the least squares method on the equation of line in space, based on the $\mathrm{n}$ set of points which determine the position of the centre of inscribed circles in the medullary canal profile (2).

$$
\sum_{i=1}^{n} e^{2}=\sum_{i=1}^{n}\left\{A x_{i}+B y_{i}+C-z_{i}\right\}^{2}
$$

The femoral neck axis is described by the line that determines the line of the femoral neck starting from the axis centre of the femoral head. One of the most effective methods for defining this rule is based on defining a cone that approximates the set of points on the femoral neck (Fig. 7).

The position of the prosthesis support in the femur describes the geometry of the endoprosthesis body at the transition from the medial to the proximal segment. The shape, dimensions and orientation of this part of the endoprosthesis body is directly dependent on the type of surgical method of removing the head and the femoral neck.

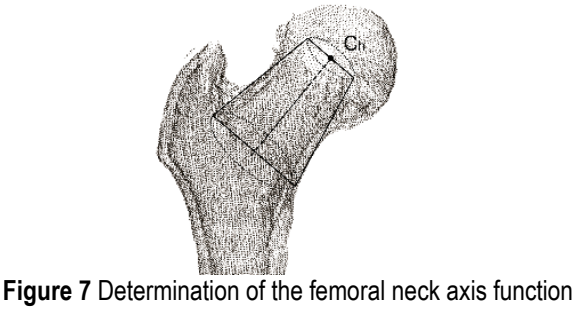

\subsubsection{Parameters of Orientation}

The geometric characteristics of the pelvic region after the incorporation of endoprosthesis significantly depend on the implants orientation in the femoral bone [22]. For the endoprosthesis design process, the angles which define the position of the head and neck in relation to the body of the femur are of great importance: cervicodiaphyseal (CCD) and anteversion (A) angle (Fig. 8).

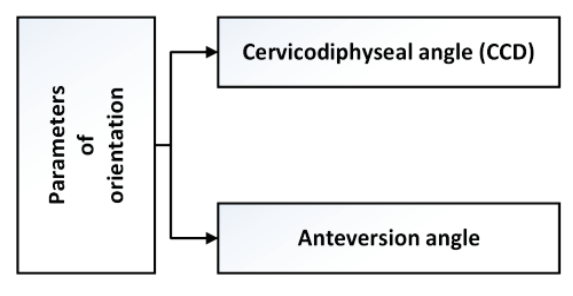

Figure 8 Parameters of orientation

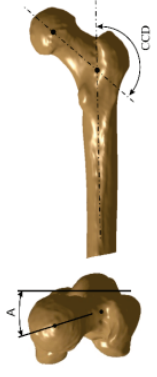

The CCD (cervicodiphyseal) angle determines the incline of the neck towards the body of the femur and is calculated based on their axes [23].

The angle of anteversion (A in Figure 8) is defined as the angle between the axis of the femoral neck and the coronal plane [24].

\subsubsection{Parameters of Shape of Endoprosthesis Body}

The developed methodology for describing the geometry of the endoprosthesis body uses a modified general mathematical model of endoprosthesis [25], in which two complex surfaces described by non-uniform rational polynomial B spline (NURBS) surfaces are utilised to define the shape of the femoral part of the implants.

The distal part of the femoral segment of endoprosthesis is of simpler structure due to the fact that the anatomical axis of the femur follows a linear trend.

The second element of the polynomial surface is based on a closed polynomial curve of the sixth order:

$$
P(t)=\frac{\sum_{i=0}^{6} \sum_{j=0}^{2} B_{i, p}^{6}(t) B_{j, p}^{2}(t) w_{i j} P_{i} P_{j}}{\sum_{i=0}^{6} \sum_{j=0}^{2} B_{i, p}^{6}(t) B_{j, p}^{2}(t) w_{i j}}
$$

The medial part of the endoprosthesis is also described by the NURBS surface where the axis of the endoprosthesis neck is depicted by a polynomial whose tangent in the end portion corresponds to the femoral neck axis equation. 


$$
P(t)=\frac{\sum_{i=0}^{6} \sum_{j=0}^{4} B_{i, p}^{6}(t) B_{j, p}^{4}(t) w_{i j} P_{i} P_{j}}{\sum_{i=0}^{6} \sum_{j=0}^{4} B_{i, p}^{6}(t) B_{j, p}^{4}(t) w_{i j}}
$$

Utilisation of the exact mathematical description of the femoral part of endoprosthesis provides flexibility of geometry which enables: obtaining different endoprosthesis sections and automatic control of endoprosthesis shape parameters according to the shape and structure of the medullary canal of the observed femur.

The remaining parameters of the endoprosthesis shape refer to the proximal segment of the endoprosthesis body, which, in practice, exist in several variants. Their geometry is based on geometric models in which the parameters determine the shape and dimensions of the supporting part $\mathrm{A}$, the cone $\mathrm{B}$ and the artificial femoral neck C (Fig. 9).

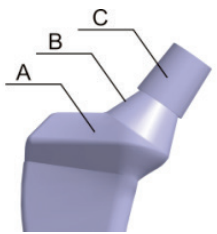

Figure 9 Proximal segment of the endoprosthesis

\section{RESULTS}

The possibilities of applying a certain methodology in the product development process are directly related to its suitability for application under different conditions. Accordingly, the evaluation of the proposed methodology for the design of endoprosthesis can be accomplished through a series of activities aimed at:

(1) Assessing the flexibility to different forms of input information,

(2) Evaluation of the success of establishing the desired geometry of the pelvis region,

(3) Determination of the benefits for application in program systems for computer supported design,

(4) Assessing the suitability for further improvements,

(5) Possibility for automated design process.

For the purposes of evaluating the methodology of designing the body of hip joint endoprosthesis software has been developed which enables the design of the endoprosthesis body and the selection of the acetabular component. The software was implemented by utilisation of Catia V5r21 Automation subsystem that enables the formation of own functions for defining and manipulating geometric shapes in product development processes. In this way, it is possible to use the basic software function for the modelling, importing and processing of diagnostic images (spatial/tomographic and planar) in the application of own methodology of prosthesis design.

The software is realised as a special workbench that contains the functions necessary for the development of endoprostheses categorised into five toolbars:

- Input information,

- Definition of location and overall dimensions of the endoprosthesis,

- Definition of orientation,

- Definition of shape of the endoprosthesis,

- Analysis of the designed solution.
The geometry of endoprosthesis is defined by surface modeling, after which it was transformed into a solid model for further design purposes.

The distal part is described by six contour shapes defined by the closed spline functions (of the sixth order), with all the control points ( $\mathrm{x}$ and $\mathrm{y}$ coordinates) defined by the parameters and controlled by the relationships that characterise the curve. The guiding curve, which defines the surface conductors in this section, is described by an open polynomial of the second order.

The shape of the medial part is defined by six contour forms also described by the closed spline functions (of the sixth order) whereby the guiding curve is a fourth-oder polynomial. The entire femoral part of endoprosthesis (distal and medial) was obtained by forming a common multi-sections surface containing the said profiles guided by the resulting curve attained by integration of the partial guiding curves. The surface thus obtained is added to the proximal part of the femur model which is defined by the surface forms, deternined by the standard of the bond between the artificial head of the femur and the body of endoprosthesis, as well as additional shapes conditioned by the requirements of the technology of manufacturing and assembly into the body (Fig. 10).

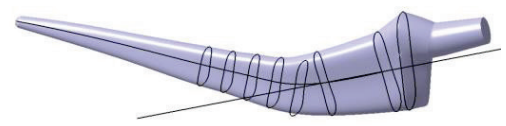

Figure 10 Graphical definition of endoprosthesis body

In order to evaluate individual activities that are implemented according to the proposed methodology, all functions of the software are realised independently, in the form of special commands (Fig. 11).

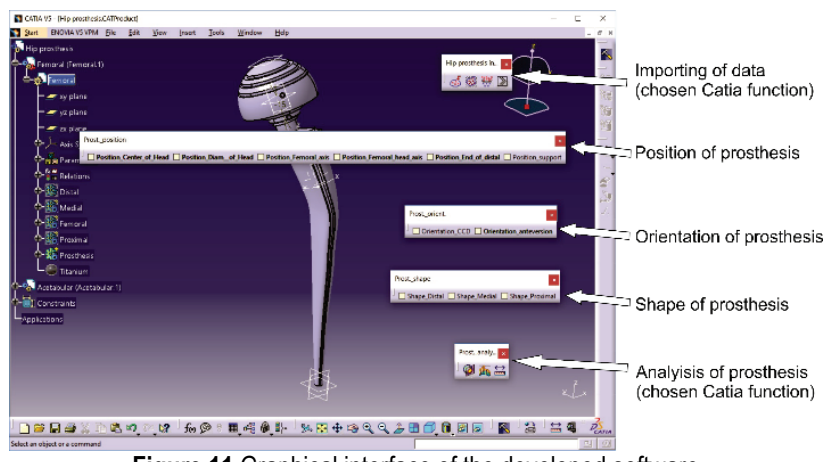

Figure 11 Graphical interface of the developed software

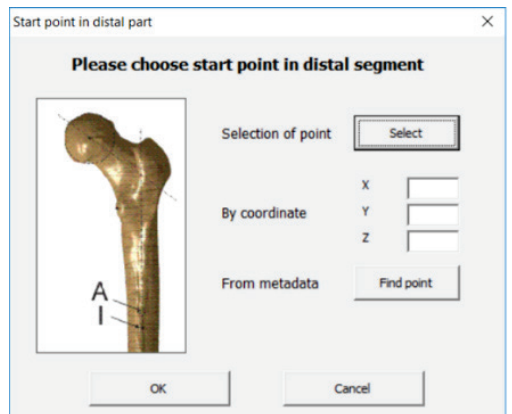

Figure 12 Dialog window for defining the location of the endoprosthesis

Functions for defining the location of the geometric elements of the endoprosthesis body in the femur are 
realised in such a way that the user is able to choose between: selection of points, input of coordinates or automated location calculation (Fig. 12).

In the process of evaluation of the methodology, ten endoprostheses were designed based on the images of different patients. In this process, different types of diagnostic images were used. Tab. 1 provides the basic characteristics of the diagnostic images used in this study.
Verification of the methodology by designing endoprostheses was realised in three phases. These phases are:

- Partial design based on planar diagnostic images,

- Endoprosthesis development by manual defining of the endoprosthesis parameters for a cloud of points that describes the femoral bone and

- $\quad$ Automated endoprosthesis design by using diagnostic images.

Table 1 Diagnostic images data

\begin{tabular}{|c|c|c|c|c|c|c|}
\hline Image label & Method & Number of images & Resolution (Pixel) & Depth (bit) & Layer offset (mm) & Device \\
\hline CT-1 & CT & 400 & $512 \times 512$ & 16 & 1 \\
\hline CT-2 & CT & 400 & $512 \times 512$ & 16 & 1 \\
\hline CT-3 & CT & 1624 & $512 \times 512$ & 16 & 0.8 \\
\hline CT-4 & CT & 1121 & $512 \times 512$ & 16 & 1 \\
\hline CT-5 & CT & 461 & $512 \times 512$ & 16 & GDCM \\
\hline MRI-6 & MRI & 48 & $336 \times 512$ & 16 & 1.5 \\
\hline MRI-7 & MRI & 192 & $320 \times 316$ & 16 & 1.5 \\
\hline DX-8 & D X-Ray & 1 & $2324 \times 2896$ & 16 & 0.5 \\
\hline DX-9 & D X-Ray & 1 & $2232 \times 2568$ & 16 & 0.143 \\
\hline DX-10 & D X-Ray & 1 & $2224 \times 2720$ & 16 & Siemens Sensation64 Sensation64 & Viemens Trio Tim \\
\hline
\end{tabular}

The initial phase of the verification is implemented by means of planar diagnostic images obtained by a digital Xray apparatus. Based on the digital planar images, it is possible to determine the most of the location parameters of the endoprosthesis (position of the centre and the diameter of the femoral head, the axis of the body and the neck of the femur in the projection of the measurement and the location of the end of the distal part of the endoprosthesis) as well as the individual parameters of orientation (CCD angle, and in case of multi-planar imaging - anteversion angle). The RADIAnt Dicom Viewer version 4.0.3 software was used for their determination. Images analysis demonstrates three geometric models of endoprosthesis (Fig. 13).
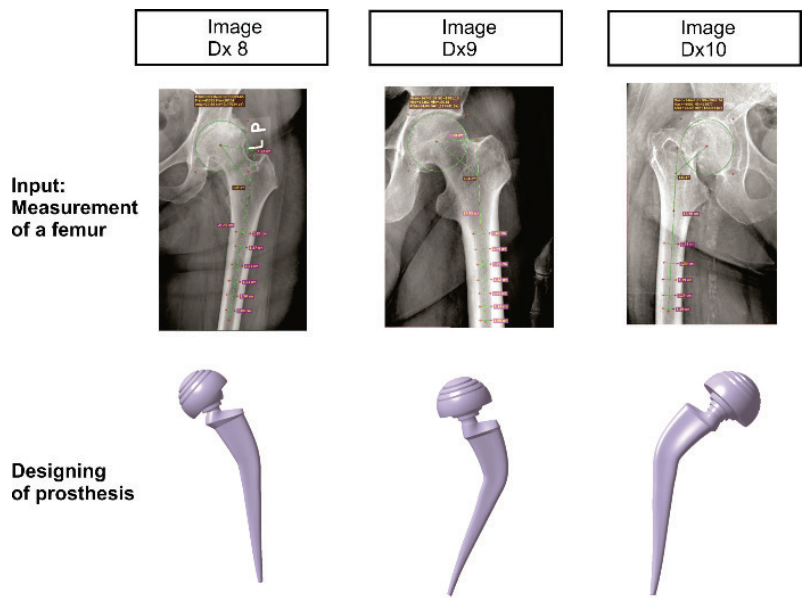

Results
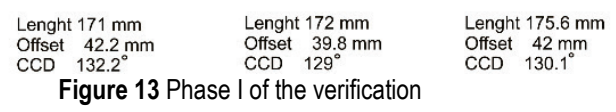

Offset $42 \mathrm{~mm}$

As a result, the most important geometric parameters of the designed endoprostheses are given at the bottom of Fig. 13.

By analysing the results, it can be concluded that the values shown in Fig. 13 should be taken with a certain reservation, since the accuracy of the measurement depends on several factors related to the imaging process and the characteristics of the device.
The second phase of the verification includes the characterisation of endoprosthesis based on the manually entered parameters of location and orientation determined by the processing of tomographic images. Input information is in the form of a cloud of points obtained by processing diagnostic images. In the verification process, a program system developed in [26] was used for obtaining the cloud of points. The design process was realised interactively, using a dialog supported correction of geometry parameters of endoprosthesis and visual integration into the geometric shape of the femur. This procedure was carried out on four diagnostic images, with two endoprosthesis bodies created by a Fused Deposition Modelling (FDM) method in order to visually control the correlation between the entered parameters (Fig. 14).

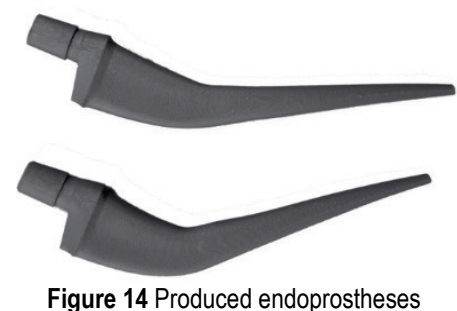

Following the definition of endoprostheses and the formation of their assembly with the reconstructed femur, the application of the CATIA program system determined the following values: femoral head diameter, neck axis, femur body axis, offset, CCD angle and angle of anteversion.

The control of the obtained values was done by comparing the acquired values with the parameters measured on the images in the 3D Doctor 4.2 software.

The error values are shown in Fig. 15 (in brackets) alongside the geometric parameters of endoprostheses obtained by design.

The obtained results indicate the advantages of applying this procedure in the prosthesis design as well as the possibilities of application in combination with commercial software for diagnostic images processing. 


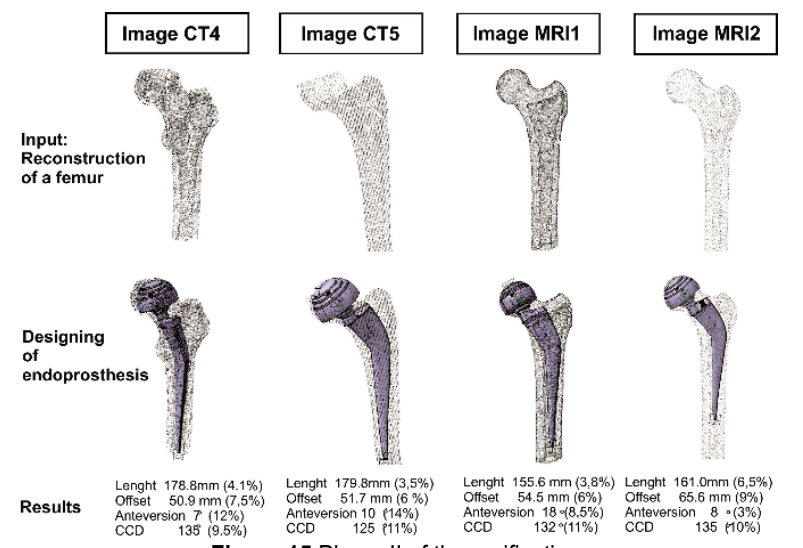

Figure 15 Phase II of the verification

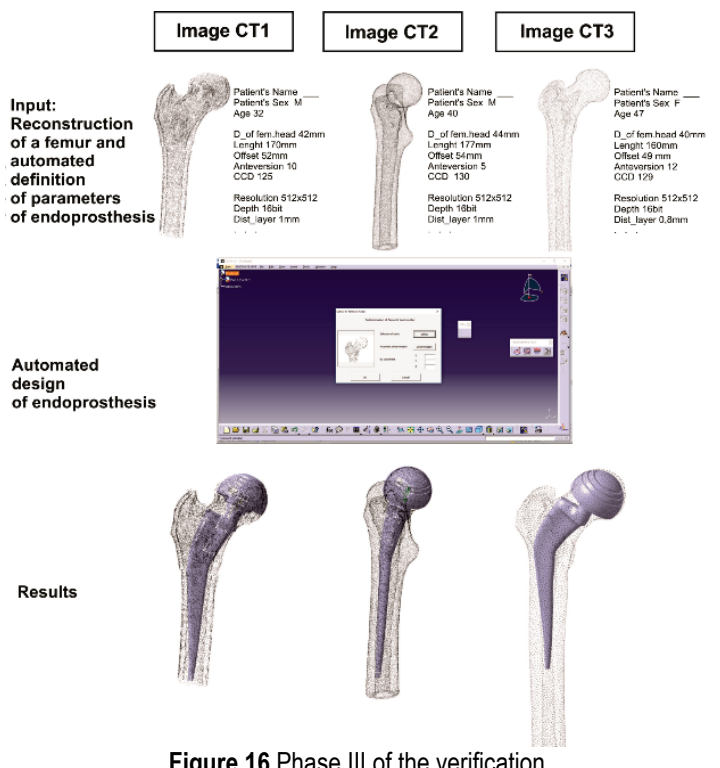

The final phase of verification involves the design of endoprostheses based on tomographic images by utilising an automated procedure for defining geometry. Input information was obtained by automatic acquisition of endoprosthesis parameters. This involves the external and internal geometry of the diseased bone in the form of a matrix of coordinates of the boundary points on the contour and the processing in order to determine all the essential geometric elements of femoral morphology. This information is transmitted to the endoprostheses design software in form of a matrix of femur geometry (cloud of points) and metadata with parameters which are important for defining endoprosthesis. Verification was applied to the first three CT images (Fig. 16).

As a result, three models of endoprostheses were obtained that were also physically made using the milling centre (Fig. 17a and b) and the FDM method (Fig. 17c).

This verification segment is used for analysis of the possibility of applying all parameters, as predicted by the methodology, in the appropriate design phases.

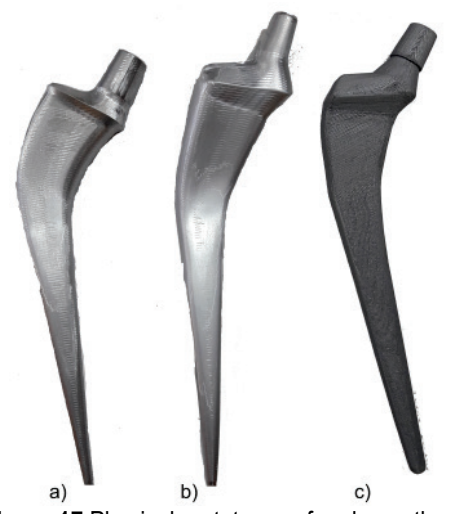

Figure 17 Physical prototypes of endoprostheses

Physically made endoprosthesis models were analysed from the standpoint of deviation of the characteristic points on the surface of the model from the nominal dimensions defined by the CAD model. Due to the complexity of endoprosthesis geometry, the analysis was performed utilising the CAD Inspection method, by scanning the prosthesis surfaces using the contact method and by comparison with the given values on the geometric model. For surfaces scanning, the coordinate measuring machine Carl Zeiss Contura G2 1000 RDS was used (Fig. 18a). PowerInspect software was used to process the results (Fig. 18b). Tab. 2 shows the results obtained by testing.

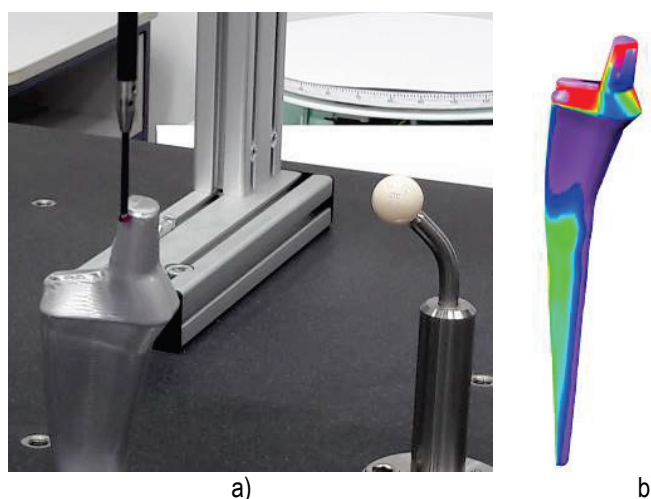

Figure 18 CAD Inspection of the endoprosthesis

Table 2 Results of verification

\begin{tabular}{|c|c|c|c|c|c|c|}
\hline Image label & Method of manuf. & Num. of points & Mean error (mm) & Min. error (mm) & Max. error (mm) & Std. deviation \\
\hline CT-1 & Milling & 8743 & 0.016 & -0.091 & 0.065 \\
\hline CT-2 & Milling & 8221 & 0.021 & -0.087 & +0.085 & +0.072 \\
\hline CT-3 & FDM & 7657 & 0.046 & -0.321 & +0.45 \\
\hline MRI-6 & FDM & 7825 & 0.052 & -0.356 & +0.205 & +0.383 \\
\hline MRI-7 & FDM & 7654 & 0.046 & -0.322 & +0.339 \\
\hline
\end{tabular}

\section{DISCUSSION}

The formation of the design process methodology of the hip joint endoprosthesis body can be seen from several perspectives. These are: scientific and commercial justification of the research, observed advantages and disadvantages of the set methodology and planned further activities on its improvement.

The basic advantage of the described methodology is the formalisation of all stages of the development and 
production of custom made implants. Additionally, defining the parameters of the endoprosthesis geometry and their association with bone morphology creates possibilities for partial or complete application of the methodology, depending on the available methods of the diseased femur imaging technique. This refers to the possibility of partial defining of implants in the application of planar imaging. There is a significant area left for its expansion with new parameters and criteria (e.g. alignment of the endoprosthesis shape with the thickness of the cement layer in the cemented endoprosthesis or the geometry of the femur in the non- cemented endoprosthesis, to name but a few).

In addition to the advantages, the analysis of the proposed methodology also pointed out to the shortcomings, elimination of which presents the base for further work in this study area. One of the shortcomings is the dependence of the design results on the factors determined by the diagnostic imaging (type and characteristics of the device, adherence to the imaging procedure, etc.).

The procedure for the development of patient-specific endoprostheses using modern engineering tools provides the possibility of designing and producing with microspatial deviations from the given values. In doing so, the process of diagnostic imaging and processing of images in real clinical conditions provides significantly less accurate input information but it does point to the improvement directions. In addition, the process of implementation of endoprostheses creates the possibility of disturbing the planned artificial hip geometry with the designer not having much impact on the ultimate goal.

The results obtained by $\mathrm{CAD}$ Inspection of the developed endoprosthes indicate that, from the engineering point of view, there are relatively large deviations from the nominal values. These values are significantly higher in prototypes made by utilising the FDM method. This was to be expected taking into account the shrinking of the material out of which the prototype is made (ABS filament). Measured errors are within the boundaries of the research on this subject [27]. Endoprostheses made on the CNC Machining Center give significantly better results that are also within the applicability limits (taking into account the expected precision of implant making), even though the technological endoprosthesis planning has not taken into account some of the essential parameters of importance for the accuracy of complex workpieces such as the use of biocompatible materials, dynamic effects of the cutting process and tolerance in the approximation of complex surfaces on CNC machines).

Research on custom made endoprostheses has to be evaluated through the application of cost-effectiveness and the necessary time for their realisation, taking into account the development status of diagnostic techniques as well as the technology of making implants. Despite significantly higher prices, research indicates an increase in the need for patient-specific endoprostheses, primarily in the revision (an increase of $140 \%$ is expected in the US over the coming period) [28] and reconstructive procedures. The number of such procedures indicates the market existence, whose growth prospects may influence primary endoprosthesis market as well. The argument for this is the fact that the described research has proven the possibility of development (processing of diagnostic images, conceptualisation, designing, FEA analysis) and the fabrication of endoprostheses within 12-16 working hours period, which indicates the competitiveness of this kind of research.

\section{CONCLUSIONS}

The process of designing and fabrication of the hip joint endoprosthesis has been a very interesting research area which still attracts considerable engineering activities. The main reasons for such interest are the complexity of biomechanical conditions, the influencing factors on the operation success and the endoprosthesis lifetime, as well as the cost-effectiveness of implant production.

The proposed methodology for the endoprostheses design is part of the authors' research on the subject of implants development in orthopedics. The hypotheses that were set in this research were completely fulfilled by the implementation of the geometric model and the methodology of defining its parameters within CAD environment in the form of developed software, as well as by creation of physical prototypes and CAD Inspection which confirms the possibilities of managing geometric parameters by using existing and introducing new correlations with the desired factors.

In addition to the advantages, the conducted research also pointed to the shortcomings of the described approach, as it is necessary to include the aforementioned parameters (tribological and technological) in the software and correlations, as well as additional parameters related to the operative procedures for the endoprostheses implantation and bone preparation. All of this points to the complexity of the problem and the necessity of further multidisciplinary research within this area.

\section{Acknowledgements}

This work was supported by the Ministry of Education, Science and Technological Development of the Republic of Serbia, under Grant No. TR 35025.

\section{REFERENCES}

[1] Pivec, R., Johnson, A. J., Mears, S. C., \& Mont, M. A. (2012). Hip arthroplasty. The Lancet, 380(9855), 1768-1777. https://doi.org/10.1016/S0140-6736(12)60607-2

[2] Culliford, D., et al. (2015). Future projections of total hip and knee arthroplasty in the UK: results from the UK Clinical Practice Research Datalink. Osteoarthritis and Cartilage, 23(4), 594-600. https://doi.org/10.1016/j.joca.2014.12.022

[3] Levadnyi, I., Awrejcewicz, J., Goethel, M. F., \& Loskutov, A. (2017). Influence of the fixation region of a press-fit hip endoprosthesis on the stress-strain state of the "boneimplant" system. Computers in Biology and Medicine, 84, 195-204. https://doi.org/10.1016/j.compbiomed.2017.03.030

[4] Čolić, K., Sedmak, A., Legweel, K., Milošević, M., Mitrović, N., Mišković, Ž., \& Hloch, S. (2017). Experimental and numerical research of mechanical behaviour of titanium alloy hip implant. Tehnički vjesnik, 24(3), 709-713.

[5] Goodnough, L. H., et al. (2018). Obesity Is Independently Associated With Early Aseptic Loosening in Primary Total 
Hip Arthroplasty. The Journal of Arthroplasty, 33(3), 882886. https://doi.org/10.1016/j.arth.2017.09.069

[6] Griza, S., et al. (2008). Fatigue failure analysis of a specific total hip prosthesis stem design. International Journal of Fatigue, 30(8), 1325-1332. https://doi.org/10.1016/j.jfatigue.2007.11.005

[7] Baxmann, M., Pfaff, A. M., Schilling, C., Grupp, T. M., Morlock, M. M. (2017). Biomechanical Evaluation of the Fatigue Performance, the Taper Corrosion and the Metal Ion Release of a Dual Taper Hip Prosthesis under Physiological Environmental Conditions. Biotribology, 12, 1-7. https://doi.org/10.1016/j.biotri.2017.07.002

[8] Fan, N. \& Chen, G. X. (2012). Numerical study of squeaking suppresses for ceramic-on-ceramic hip endoprosthesis. Tribology International, 48, 172-181. https://doi.org/10.1016/j.triboint.2011.11.023

[9] Chen, Q. \& Thouas, G. A. (2015). Metallic implant biomaterials. Materials Science and Engineering: $R$ : Reports, 87, 1-57. https://doi.org/10.1016/j.mser.2014.10.001

[10] Oksiuta, Z., Mazurkiewicz, Z., Romanczuk, E., Andrzejewicz, M., \& Krawczynska, A. (2017). Analysis of microstructural aspects of a hip stem failure made of the REX 734 stainless steel. Engineering Failure Analysis, 72, 1-10. https://doi.org/10.1016/j.engfailanal.2016.11.012

[11] Yakupov, R. R., et al. (2017). Studies of the Adhesive Component of Friction in Hip Joint Endoprostheses. Biomedical Engineering, 51(4), 271-274. https://doi.org/10.1007/s10527-017-9729-0

[12] Shuster, L. S., Chertovskikh, S. V., Minasov, B. S., Yakupov, R. R., Emaev, I. I. (2016). Tribological characteristics of hip joint endoprostheses. Journal of Friction and Wear, 37(1), 1-6. https://doi.org/10.3103/S1068366616010165

[13] Hua, Z., et al. (2016). Analysis of the Friction-Induced Squeaking of Ceramic-on-Ceramic Hip Prostheses Using a Pelvic Bone Finite Element Model. Tribology Letters, 61(3), 26. https://doi.org/10.1007/s11249-016-0644-4

[14] Jun, Y. \& Choi, K. (2010). Design of patient-specific hip implants based on the 3D geometry of the human femur. Advances in Engineering Software, 41(4), 537-547. https://doi.org/10.1016/j.advengsoft.2009.10.016

[15] Liu, D., Hua, Z., Yan, X., \& Jin, Z. (2016). Design and biomechanical study of a novel adjustable hemipelvic prosthesis. Medical Engineering \& Physics, 38(12), 14161425. https://doi.org/10.1016/j.medengphy.2016.09.017

[16] Tsukada, S. \& Wakui, M. (2016). A Case Series of Total Hip Arthroplasty Using Cementless Hip Stem Customized for Patients of a Specific Race: 10- to 15-Year Results. The Journal of Arthroplasty, 31(1), 190-193. https://doi.org/10.1016/j.arth.2015.07.036

[17] Almeida, D. F., et al. (2016). Fully automatic segmentation of femurs with medullary canal definition in high and in low resolution CT scans. Medical Engineering \& Physics, 38(12), 1474-1480. https://doi.org/10.1016/j.medengphy.2016.09.019

[18] Park, B.-K., Bae, J.-H., Koo, B.-Y., \& Kim, J. J. (2014). Function-based morphing methodology for parameterizing patient-specific models of human proximal femurs. Computer-Aided Design, 51, 31-38. https://doi.org/10.1016/j.cad.2014.02.003

[19] Popovich, A. A., et al. (2016). Use of Additive Techniques for Preparing Individual Components of Titanium Alloy Joint Endoprostheses. Biomedical Engineering, 50(3), 202205. https://doi.org/10.1007/s10527-016-9619-x

[20] Boyle, C. \& Kim, Y. (2011). Comparison of different hip prosthesis shapes considering micro-level bone remodeling and stress-shielding criteria using three-dimensional design space topology optimization. Journal of Biomechanics, 44(9), 1722-1728.

https://doi.org/10.1016/j.jbiomech.2011.03.038
[21] Bert, J. M. (1996). Custom total hip arthroplasty. The Journal of Arthroplasty, 11(8), 905-915. https://doi.org/10.1016/S0883-5403(96)80131-8

[22] Widmer, K. H. \& Zurfluh, B. (2004). Compliant positioning of total hip components for optimal range of motion. Journal of Orthopaedic Research, 22(4), 815-821. https://doi.org/10.1016/j.orthres.2003.11.001

[23] Traina, F., et al. (2009). Sex differences in hip morphology: is stem modularity effective for total hip replacement? $J$ Bone Joint Surg Am, 91(Suppl 6), 121-128. https://doi.org/10.2106/JBJS.I.00533

[24] Park, K. K., Tsai, T.-Y., Dimitriou, D., \& Kwon, Y.-M. (2015). Utility of Preoperative Femoral Neck Geometry in Predicting Femoral Stem Anteversion. The Journal of Arthroplasty, 30(6), 1079-1084. https://doi.org/10.1016/j.arth.2015.01.016

[25] Tabaković, S., Zeljković, M., \& Živković, A. (2014). General parametric model of the body of the total hip endoprosthesis. Acta Polytechnica Hungarica, 11(1), 227246.

[26] Tabakovic, S., Zeljkovic, M., \& Milojevic, Z. (2014). Automated acquisition of proximal femur morphological characteristics. Measurement Science Review, 14(5), 285293. https://doi.org/10.2478/msr-2014-0039

[27] Sood, A. K., Ohdar, R. K., \& Mahapatra, S. S. (2009). Improving dimensional accuracy of Fused Deposition Modelling processed part using grey Taguchi method. Materials \& Design, 30(10), 4243-4252. https://doi.org/10.1016/..matdes.2009.04.030

[28] Kurtz, S., Ong, K., Lau, E., Mowat, F., \& Halpern, M. (2007). Projections of primary and revision hip and knee arthroplasty in the United States from 2005 to 2030. J Bone Joint Surg Am, 89(4), 780-785.

\section{Contact information:}

Dr. Slobodan TABAKOVIĆ, Associate Professor

(Corresponding author)

University of Novi Sad,

Faculty of Technical Sciences,

Trg Dositeja Obradovića 6

21000 Novi Sad, Serbia

E-mail: tabak@uns.ac.rs

\section{Dr. Milan ZELJKOVIĆ, Full Professor}

University of Novi Sad,

Faculty of Technical Sciences,

Trg Dositeja Obradovića 6,

21000 Novi Sad, Serbia

E-mail: milanz@uns.ac.rs

\section{Dr. Zoran MILOJEVIĆ, Associate Professor}

University of Novi Sad,

Faculty of Technical Sciences,

Trg Dositeja Obradovića 6,

21000 Novi Sad, Serbia

E-mail: zormil@uns.ac.rs

Dr. Aleksandar ŽıVKOVIĆ, Asistant Professor

University of Novi Sad,

Faculty of Technical Sciences,

Trg Dositeja Obradovića 6

21000 Novi Sad, Serbia

E-mail: acoz@uns.ac.rs 